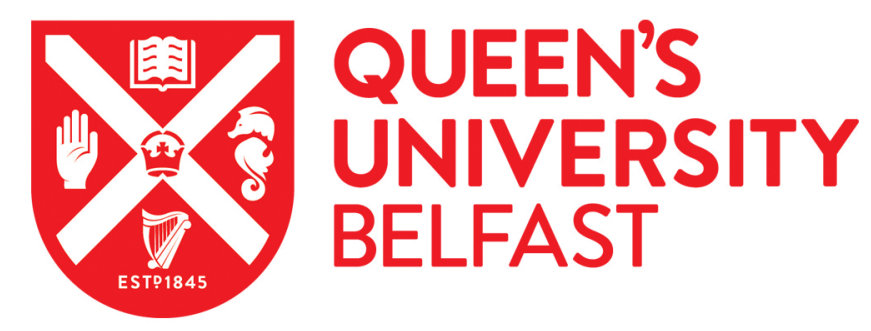

\title{
Selective Ion Acceleration by Intense Radiation Pressure
}

Mcllvenny, A., Doria, D., Romagnani, L., Ahmed, H., Booth, N., Ditter, E-J., Hicks, G. S., Ettlinger, O. C., Martin, P., Scott, G. G., Williamson, S. D. R., Macchi, A., McKenna, P., Najmudin , Z., Neely, D., Kar, S., \& Borghesi, M. (2021). Selective Ion Acceleration by Intense Radiation Pressure. Physical Review Letters, 127(19), [194801]. https://doi.org/10.1103/PhysRevLett.127.194801

Published in:

Physical Review Letters

Document Version:

Publisher's PDF, also known as Version of record

Queen's University Belfast - Research Portal:

Link to publication record in Queen's University Belfast Research Portal

Publisher rights

(C) 2021 American Physical Society

This work is made available online in accordance with the publisher's policies. Please refer to any applicable terms of use of the publisher.

\section{General rights}

Copyright for the publications made accessible via the Queen's University Belfast Research Portal is retained by the author(s) and / or other copyright owners and it is a condition of accessing these publications that users recognise and abide by the legal requirements associated with these rights.

Take down policy

The Research Portal is Queen's institutional repository that provides access to Queen's research output. Every effort has been made to ensure that content in the Research Portal does not infringe any person's rights, or applicable UK laws. If you discover content in the Research Portal that you believe breaches copyright or violates any law, please contact openaccess@qub.ac.uk. 


\title{
Selective Ion Acceleration by Intense Radiation Pressure
}

\author{
A. McIlvenny $\odot,{ }^{1, \dagger}$ D. Doria $\odot,{ }^{1,2}$ L. Romagnani®$\odot,{ }^{1,3}$ H. Ahmed $\odot,{ }^{1,4}$ N. Booth, ${ }^{4}$ E. J. Ditter $\odot,{ }^{5}$ O. C. Ettlinger, \\ G. S. Hicks $\odot,^{5}$ P. Martin $\odot,{ }^{1}$ G. G. Scott, ${ }^{4}$ S. D. R. Williamson, ${ }^{6}$ A. Macchi $\odot,{ }^{7,8}$ P. McKenna $\odot,{ }^{6}$ Z. Najmudin $\odot,{ }^{5}$ D. Neely, ${ }^{4, *}$ \\ S. Kar $\oplus^{1}$ and M. Borghesi $\oplus^{1, \$}$ \\ ${ }^{1}$ Centre for Plasma Physics, Queens University Belfast, Belfast BT7 1NN, United Kingdom \\ ${ }^{2}$ Extreme Light Infrastructure (ELI-NP) and Horia Hulubei National Institute for $R \& D$ in Physics and Nuclear Engineering \\ (IFIN-HH), 30 Reactorului Street, 077125 Magurele, Romania \\ ${ }^{3}$ LULI-CNRS, Ecole Polytechnique, CEA, Universit Paris-Saclay, F-91128 Palaiseau cedex, France \\ ${ }^{4}$ Central Laser Facility, Rutherford Appleton Laboratory, Oxfordshire OX11 OQX, United Kingdom \\ ${ }^{5}$ The John Adams Institute for Accelerator Science, Blackett Laboratory, Imperial College London, London SW7 2BZ, United Kingdom \\ ${ }^{6}$ SUPA, Department of Physics, University of Strathclyde, Glasgow G4 ONG, United Kingdom \\ ${ }^{7}$ Istituto Nazionale di Ottica, Consiglio Nazionale delle Ricerche (CNR/INO), research unit Adriano Gozzini, Pisa 56124, Italy \\ ${ }^{8}$ Dipartimento di Fisica Enrico Fermi, Università di Pisa, Pisa 56127, Italy
}

(Received 4 January 2021; revised 20 August 2021; accepted 9 September 2021; published 1 November 2021)

\begin{abstract}
We report on the selective acceleration of carbon ions during the interaction of ultrashort, circularly polarized and contrast-enhanced laser pulses, at a peak intensity of $5.5 \times 10^{20} \mathrm{~W} / \mathrm{cm}^{2}$, with ultrathin carbon foils. Under optimized conditions, energies per nucleon of the bulk carbon ions reached significantly higher values than the energies of contaminant protons $(33 \mathrm{MeV} /$ nucleon vs $18 \mathrm{MeV}$ ), unlike what is typically observed in laser-foil acceleration experiments. Experimental data, and supporting simulations, emphasize different dominant acceleration mechanisms for the two ion species and highlight an (intensity dependent) optimum thickness for radiation pressure acceleration; it is suggested that the preceding laser energy reaching the target before the main pulse arrives plays a key role in a preferential acceleration of the heavier ion species.
\end{abstract}

DOI: 10.1103/PhysRevLett.127.194801

Laser-driven ion beams have gained significant scientific interest owing to their unique characteristics, such as ultrashort duration, high flux, and ultralow emittance $[1,2]$; this makes them a promising candidate for use in a range of applications [3,4]. While sheath acceleration mechanisms such as target normal sheath acceleration (TNSA) offer these properties, they suffer from a relatively slow scaling of ion energies with laser intensity limiting the applicative potential of these beams [5]. Furthermore, the dominant acceleration of protons typically shields and prevents acceleration of heavier ions [6], such as carbon, which also have significant applicative interest. Recently, mechanisms acting during the irradiation of ultrathin foils, such as radiation pressure acceleration (RPA), or acceleration in relativistically induced transparency (RIT) regimes have attracted significant attention $[7,8]$. A number of numerical studies have predicted that RPA will intrinsically dominate at intensities greater than $10^{22} \mathrm{~W} / \mathrm{cm}^{2}$ with potential to produce monoenergetic beams of bulk target ions [9-15]; in particular, the "light sail" (LS) mode of RPA offers quadratic scaling of ion energies with laser fluence or a reduced target areal density, highlighting it as a promising route towards the production of high energy, high quality ion beams $[8,11]$. Numerical and experimental work has shown that this regime can be accessed at currently available intensities by using a circularly polarized $(\mathrm{CP})$ pulse to reduce the electron temperature and allow RPA features to emerge from (or dominate over) sheath acceleration effects [13,16-22].

In this Letter, we report on the experimental verification of an (intensity dependent) optimum target thickness for bulk ion acceleration via LS-RPA, as well as on the selective acceleration, at the optimum thickness, of bulk (carbon) ions to significantly higher energies than contaminant protons, whose energy is instead seen to drop substantially at this optimum thickness. This is the opposite of what is normally observed in multispecies ion acceleration from thin foils, where protons typically gain energy more efficiently during the acceleration process, thanks to the favorable $Z / A$ ratio $[16,17,23,24]$. Multidimensional particle in cell (PIC) simulations, carried out for the conditions of the experiment, indicate that by exploiting the pulse's unavoidable preceding intensity (the coherent contrast, usually a result of imperfect pulse compression [25]), proton contaminants can be displaced from the masslimited target allowing the remaining carbon dominated target bulk to be accelerated by LS-RPA. The understanding provided in this Letter highlights routes for controllable, selective acceleration of a single ion species in the LS-RPA regime by tailoring the coherent contrast or 
by using a separate prepulse. This approach will be an important step towards the development of versatile, high energy ion sources, and will facilitate a range of applications requiring single-species ion beams accelerated from the target bulk (an example being high dose-rate carbon radiobiology [26]).

The experiment was carried out using the GEMINI laser facility at the Rutherford Appleton Laboratory U.K. A 40 fs FWHM laser pulse, with central wavelength $\lambda=800 \mathrm{~nm}$ was used, and temporally cleaned by a double plasma mirror arrangement $[27,28]$ producing a contrast on the order of $10^{-6}$ at $1 \mathrm{ps}$ and $10^{-14}$ at $100 \mathrm{ps}$ which is sufficient to prevent significant premature expansion of the targets before the plasma mirrors are activated [29]. The pulse was focused using an $f / 2$ parabola at normal incidence producing a $3 \mu \mathrm{m}$ FWHM spot with $\sim 6 \mathrm{~J}$ on target and a peak intensity of $5.5 \times 10^{20} \mathrm{~W} / \mathrm{cm}^{2}$.

The main diagnostics were three Thomson parabola spectrometers (TPSs) placed at $-4^{\circ}, 0^{\circ}$, and $9^{\circ}$ with respect to the target normal, each coupled to a microchannel plate (MCP)-CCD system to instantaneously record the ion spectra. Amorphous carbon targets (density of $\sim 2 \mathrm{~g} / \mathrm{cm}^{3}, \mathrm{n}_{e}=350 \mathrm{n}_{c}$ where $\mathrm{n}_{e}$ and $\mathrm{n}_{c}$ are the electron density and plasma critical density, respectively) with thickness in the range $2-100 \mathrm{~nm}$ were irradiated to match the optimal LS parameters; an optimal areal density $(\sigma)$ equivalent to a target thickness of $\sim 10 \mathrm{~nm}$ at this electron density is required for the dimensionless laser amplitude $a_{0} \approx 13$ [11]. The laser polarization could be controlled with a $\lambda / 4$ wave plate placed in the collimated beam between the plasma mirrors and the parabola.

The maximum energies for the two main species in the spectra $\left(\mathrm{C}^{6+}\right.$ and $\left.\mathrm{H}^{+}\right)$as recorded on the $0^{\circ}$ TPS for $\mathrm{CP}$ shots are shown in Fig. 1. The data highlights the presence of an optimal thickness at $15 \mathrm{~nm}$, where the $\mathrm{C}^{6+}$ energies reach up to $33 \mathrm{MeV} /$ nucleon $(\sim 400 \mathrm{MeV})$. A notable feature of the data is the fact that, under the conditions where the maximum carbon energies are obtained $(15 \mathrm{~nm}$ and $\mathrm{CP})$, a local minimum (18 MeV) is obtained for the proton energies. The intensity dependence of the maximum energy for the two ion species at the optimum thickness of $15 \mathrm{~nm}$ is shown in Fig. 2(a). While $\mathrm{C}^{6+}$ scales favorably with intensity $\left(E_{C^{6+}} \propto I^{1.2 \pm 0.2}\right), \mathrm{H}^{+}$follows a much slower trend. This behavior is not observed for linear polarization where there is no clear peak in carbon energies and consistently higher proton energies are observed for the same target thicknesses.

The preferential acceleration of the heavier species over protons as observed here is atypical of the acceleration mechanisms known to act on multispecies targets. In acceleration schemes such as TNSA, electrons set up a sheath field which will preferentially accelerate protons over other ion species due to their higher $Z / A$ ratio, always resulting in higher energies for protons; this has also been

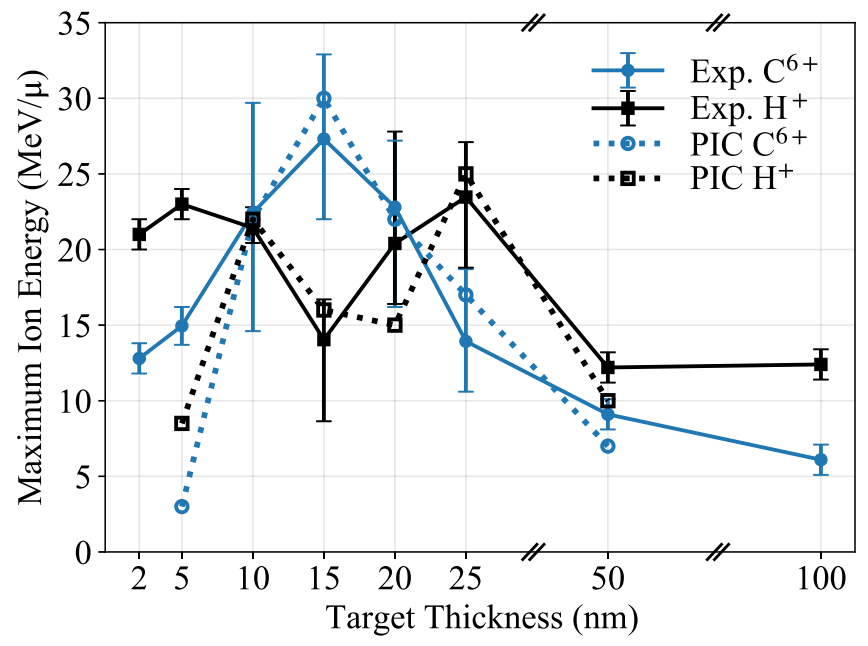

FIG. 1. Maximum ion energy from $0^{\circ}$ TPS for $\mathrm{C}^{6+}$ (solid line and filled circles) and $\mathrm{H}^{+}$(solid line and filled squares) for $\mathrm{CP}$. 2D PIC simulations are dotted lines with the same markers but empty. Errors bars represent the fluctuations in the measured maximum energy due to shot to shot variation in laser energy for intensities greater than $4 \times 10^{20} \mathrm{~W} / \mathrm{cm}^{2}$ throughout the experiment.

observed in the RIT regime [7,30,31] as well as in LS-RPA $[13,16,17,21,23,32]$.

The observations at $15 \mathrm{~nm}$ therefore suggest that during the interaction for the optimized LS-RPA conditions, different acceleration processes act on the two species. In order to test this hypothesis, we have carried out supporting simulations with the EPOCH PIC code [33] for two cases: with and without the coherent contrast.

The first case considered a flat foil irradiated at normal incidence by an idealized $40 \mathrm{fs}, 3 \mu \mathrm{m}$ pulse (both are Gaussian FWHM, with the simulation beginning at time $t=$ $-100 \mathrm{fs}$ so that no coherent contrast is considered). The target was composed of $\mathrm{C}^{6+}$ neutralized with electrons at a density of $350 \mathrm{n}_{c}$ with front and rear surface, $5 \mathrm{~nm}$-thick $10 \mathrm{n}_{c} \mathrm{H}^{+}$layers to simulate contaminant layers (where the $\mathrm{H}^{+}$observed in experiments usually originate from). The simulations were performed on a grid with resolution $\Delta x=5 \mathrm{~nm}, \Delta y=4 \Delta x$, and $x$ range $[-10 \mu \mathrm{m}, 30 \mu \mathrm{m}]$. The target was initially located at $x=0$, with $y$ range $[-5 \mu \mathrm{m}, 5 \mu \mathrm{m}]$ and was initialized with 200 particles per cell per species with a temperature of $10 \mathrm{keV}$. Collisions are not calculated in this case, as justified by their negligible influence at the temperature generated at this intensity. In these PIC simulations with an idealized pulse profile, the $\mathrm{H}^{+}$ energies scale similarly to carbon albeit they are $\sim 30 \%$ higher [triangles in Fig. 2(b)] and reproduce the $I^{2}$ scaling associated with LS-RPA since an idealized target at this thickness (without considering any significant preexpansion) can enter the LS stage almost instantaneously and remain opaque for the majority of the pulse duration.

A better insight into the possible causes of the different behavior of protons and carbon ions (as observed in the 

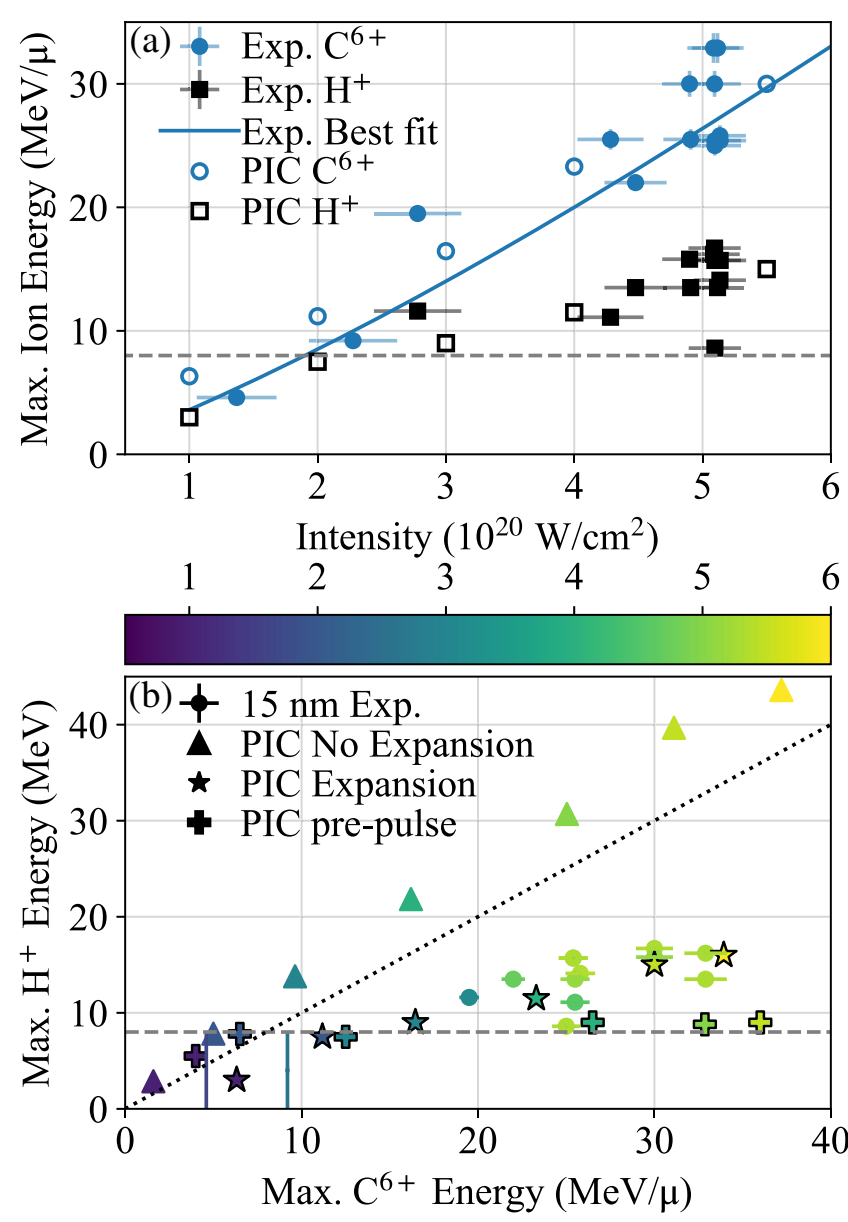

FIG. 2. (a) Maximum ion energy as a function of laser intensity for experimental measurements of $\mathrm{C}^{6+}$ (filled blue circles) and $\mathrm{H}^{+}$(filled black squares). 2D PICs with expansion included are also shown with the same markers but empty. The solid line represents the best fit line to the $\mathrm{C}^{6+}$ experimental data, $\propto I^{1.2 \pm 0.2}$. (b) Correlation between $\mathrm{C}^{6+}$ and $\mathrm{H}^{+}$maximum energies for experimental and simulation data (marker colors indicate the laser intensity). Dotted line indicates where $\mathrm{C}^{6+}$ and $\mathrm{H}^{+}$energies are equal. The dashed line at $y=8 \mathrm{MeV}$ in (a) and (b) represents the detection threshold for protons, and the maximum proton energy did not meet this threshold for the two lowest intensity shots. Crosses indicate simulations which included a prepulse with a peak intensity of $10^{17} \mathrm{~W} / \mathrm{cm}^{2}$ arriving on target 2.5 ps before the peak of the main pulse.

experimental data of Figs. 1 and 2) can be obtained by introducing a more realistic temporal profile of the laser pulse into the simulations. The plasma mirrors, used to reduce target heating, are typically activated a few ps before the main pulse as the inherent contrast level decreases, meaning that the pulse is still preceded by a short pedestal and rising edge as shown in Fig. 3. This was considered in the second set of simulations. The intensity profile is based on a third order cross-correlator scan of the pulse before any contrast enhancement from the plasma mirrors (also shown in Fig. 3). Measurements on a similar system have

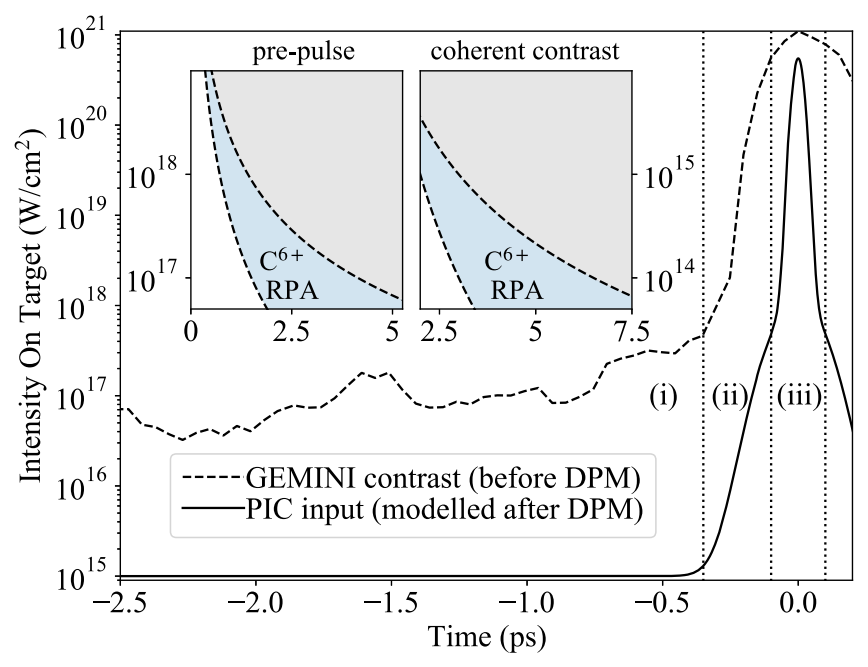

FIG. 3. Cross-correlator data of the GEMINI laser pulse profile without the double plasma mirror (DPM) arrangement in blue (dashed) alongside a modeled, contrast enhanced pulse profile used as a simulation input (solid black) and based on [34]. The pulse profiles have a peak intensity of $5.5 \times 10^{20} \mathrm{~W} / \mathrm{cm}^{2}$ and $1.1 \times 10^{21} \mathrm{~W} / \mathrm{cm}^{2}$ with and without plasma mirrors, respectively. Three annotated areas (divided by dotted black lines) mark the parts of the interaction highlighted in the text. The inset graphs show the parameter space for preferential species acceleration using a prepulse (left) and the coherent contrast (right) based on 2D PIC simulations. The intensities on the vertical axes indicate, respectively, the peak intensity of the prepulse (left frame) and the intensity of the flat pedestal (right frame), while the horizontal axes indicate the time separation between the prepulse and main pulse (left) and the start of the pedestal and the main pulse (right). The main pulse remains unchanged. The blue shaded areas indicate where preferential $\mathrm{C}^{6+}$ acceleration by RPA is observed. The white area is where $\mathrm{H}^{+}$is favored by RPA and the grey area is where $\mathrm{n}_{e}<\gamma \mathrm{n}_{c}$ and proton acceleration is favored in the RIT regime.

compared a similar cross-correlator scan with a much higher resolution technique both with and without a plasma mirror [34]. Based on this work, a truer representation of the experimental pulse profile is modeled in our simulations. The same grid is initialized in these simulations except now with ionization [35-37] and collisions [38] calculated and the target set as unionized and cold. Additional simulations with different contrast levels are shown in the Supplemental Material [39].

At the optimum thickness, the interaction can be thought of as consisting of three stages: (i) expansion, (ii) recompression, and (iii) acceleration. The low intensity pedestal is responsible for the majority of the ionization and expansion. At this stage protons, with their lighter mass, will expand faster than the carbon ions and are mainly located in the front and rear underdense plasma. The target bulk has also expanded but it remains overdense. Furthermore, the expansion of the target inevitably means the mass of the target bulk partaking in the 

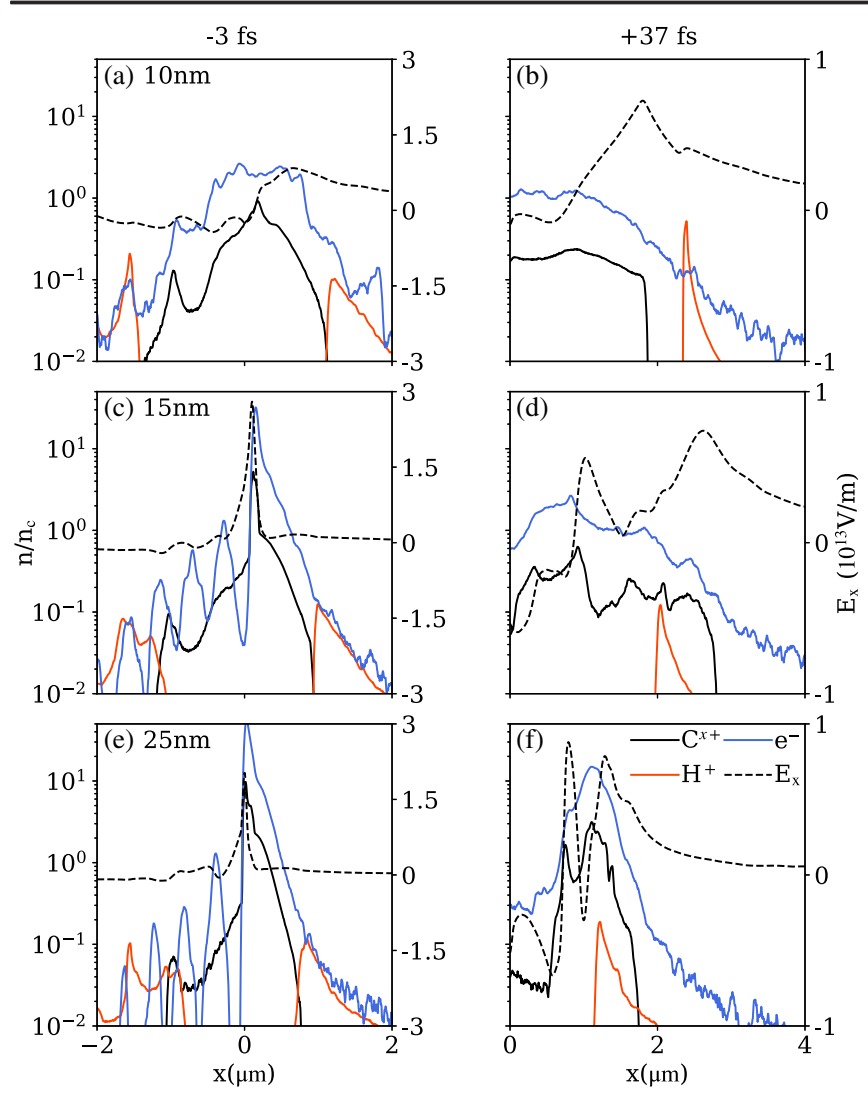

FIG. 4. Average density (left logarithmic axes) for $\mathrm{C}^{6+}$ (solid black), protons (red), and electrons (blue) for $10 \mathrm{~nm}[(\mathrm{a}),(\mathrm{b})]$, $15 \mathrm{~nm}[(\mathrm{c}),(\mathrm{d})]$, and $25 \mathrm{~nm}$ [(e),(f)] along the laser axis (averaged between $y= \pm 100 \mathrm{~nm}$ ). The right axes display the cycle-averaged longitudinal electric field ( $E_{x}$, dashed black line and linear axis). The left panels capture the interaction at close to the peak of the pulse ( $-3 \mathrm{fs}$ ) while the panels on the right refer to a time towards the end of the pulse $(+37 \mathrm{fs})$.

subsequent LS-RPA stage is reduced explaining why the experimentally observed optimal thickness is higher than that predicted theoretically [11].

Less than 1 ps before the main pulse arrives, the intensity rises to the point where the thermal pressure in the target is overcome by the radiation pressure. The laser reflects off the overdense plasma, setting up a standing wave with the incoming radiation, which increases the local electric field, and pushes electrons to the points of lower laser intensity [corresponding to the density modulations in Figs. 4(c) and 4(e) for $x<0$ ]. The laser's rising edge begins to compress the front surface which further steepens the front density gradient, reversing some of the density decrease caused by the earlier expansion; this affects only the overdense carbon plasma, as the protons are not present in significant number in this central region. When the pulse peak arrives, it interacts with a recompressed (but reduced density) plasma which is close to the optimal areal density for the incident intensity (see Supplemental Material [39]). Here the laser accelerates the carbon bulk by LS-RPA whereas proton energies will be determined by subsequent sheath acceleration, as shown in Figs. 4(c) and 4(d); as a result of these dynamics, the high energy carbon ions from the target bulk are accelerated much more efficiently than protons.

The LS-RPA phase is followed by an RIT phase which also has a role in accelerating ions. Close to the peak of the pulse, the plasma becomes relativistically transparent. Comparisons of simulations with and without this laser energy propagating through the target show that the RIT regime accounts for $\sim 37 \%$ of the final $\mathrm{C}^{6+}$ energy and $\sim 63 \%$ of the final proton energy.

The peak intensity was also varied in these simulations reproducing the experimentally observed speciesdependent scaling of the cut-off energy with laser intensity (Fig. 2). The simulations show that a reduced intensity scaling for carbon compared to the ideal $I^{2}$ dependence arises from the combination of two effects: (1) a delayed transition to LS since the initial hole boring phase must penetrate through the now expanded target; (2) an earlier transition to transparency for more intense pulses.

Achieving preferential carbon acceleration requires sufficient relative expansion of the proton species, such that protons are present only in the underdense preplasma, all while the target bulk remains relativistically overdense. As shown in the inset graphs of Fig. 3, these conditions can be accessed through the target heating induced by the coherent contrast reflected from the plasma mirrors, as in the experiment, but could also be reached by introducing a prepulse, of equal duration to the main pulse, a few ps before the pulse peak (representative of a pickoff from the main beam). The left inset panel in Fig. 3, summarizes results of 2D PIC simulations in which a $40 \mathrm{fs}$ pulse, of varying peak intensity ( $y$ axis), irradiates the target at a time ( $x$ axis) before the main beam arrives, which allows the target to expand. The parameter region where preferential carbon acceleration was observed in simulations is shown in blue. Data points from a simulation employing a $10^{17} \mathrm{~W} / \mathrm{cm}^{2}$ prepulse $2.5 \mathrm{ps}$ before the main pulse are also shown in Fig. 2(b) as an example. Additional PIC simulations were performed to explore the effect of the intensity and duration of the coherent contrast on target (see right inset panel in Fig. 3). This was done by varying the amplitude of the flat pedestal [section (i) of the pulse profile in Fig. 3] and varying the arrival time of the peak ( $x$ axis of the right-hand inset in Fig. 3) showing that a window of 3-8 ps for $10^{14} \mathrm{~W} / \mathrm{cm}^{2}$ as opposed to $2-2.5 \mathrm{ps}$ for $10^{15} \mathrm{~W} / \mathrm{cm}^{2}$ is required for preferential carbon acceleration. In general terms, if the laser contrast is better then one would need to activate the plasma mirrors earlier (by using a tighter focus on their surface) to achieve the same degree of target expansion. Both of these methods highlight routes to control the preferential acceleration of the carbon bulk.

The simulations also provide an explanation for the local minimum in the $\mathrm{H}^{+}$energies and a comparison between 
experiments and simulations is shown in Fig. 1. At the optimal thickness, the carbon bulk is accelerated much more efficiently by LS-RPA which, in turn, reduces the sheath potential at the target rear where the protons are located. The carbon ions will overtake the proton contaminants and are themselves further accelerated in the sheath [Fig. 4(d)].

For targets thicker than the optimum, the energy gain for the $\mathrm{C}^{6+}$ bulk is significantly reduced due to the increased areal density. While the transition to transparency is delayed, this does not overcome the reduced efficiency of the acceleration $\left(E_{\max } \propto \sigma^{-2}\right)[8]$. Here, sheath acceleration dominates and favors protons since the carbon bulk remains behind the proton contaminants. For targets thinner than the LS optimum, by the time the main pulse arrives, the target has expanded so much that it is relativistically transparent (where $\mathrm{n}_{e}<\gamma \mathrm{n}_{c}$, where $\gamma$ is the Lorentz factor, as seen in Fig. 4(a)). The $\mathrm{C}^{6+}$ ions can gain energy very quickly in an initial RPA phase but this is short lived as the target is too thin to sustain LS-RPA at this intensity and RIT sets in well before the peak of the pulse. This reduces the energy gain for carbons, but causes direct electron heating as the pulse propagates through the transparent plasma [Figs. 4(a) and 4(b)]. Since protons have expanded at the rear, they will experience the strongest field from this heating effect, screening the $\mathrm{C}^{6+}$ ions, and are subsequently accelerated to higher energies [31].

In conclusion, we have demonstrated, through experiments and simulations, that a moderate preexpansion of ultrathin targets can lead, via LS-RPA, to dominant acceleration of bulk carbon ions over contaminant protons. Furthermore, we present a more general case for controlling the expansion of a multispecies, ultrathin foil to selectively accelerate a single species. This can be achieved, in principle, by either modifying the activation time of the plasma mirror or by using a separate ps-timescale prepulse to controllably preheat the target as indicated by the simulations presented in this Letter. Additional opportunities for control may be achieved by modifying the pulse profile on a sub-ps timescale by tuning the third order dispersion [40] to further tailor the target density profiles prior to the arrival of the peak of the pulse. We believe that these considerations will be highly relevant and applicable to future investigations on multi-PW systems, where RPA acceleration will be a major topic of investigations. Our findings point to a promising route towards a versatile source of high energy ions with control over the preferential acceleration of a single species.

Data is available from [41].

The authors would like to thank the GEMINI staff, engineering and target fabrication groups at the CLF for their support. This work was funded by EPSRC through grant EP/K022415/1. The EPOCH code is also funded by EPSRC (grants EP/G056803/1, EP/G055165/1 and
EP/M022463/1). The authors acknowledge computing resources provided by STFC Scientific Computing Department's SCARF cluster as well Kelvin High Performance Computing cluster at Queen's University Belfast.
"Deceased.
Corresponding author. amcilvenny01@qub.ac.uk
Corresponding author. m.borghesi@qub.ac.uk

[1] A. Macchi, M. Borghesi, and M. Passoni, Ion acceleration by superintense laser-plasma interaction, Rev. Mod. Phys. 85, 751 (2013).

[2] H. Daido, M. Nishiuchi, and A. S. Pirozhkov, Review of laser-driven ion sources and their applications, Rep. Prog. Phys. 75, 056401 (2012).

[3] J. Schreiber, P. R. Bolton, and K. Parodi, Invited review article: "Hands-on" laser-driven ion acceleration: A primer for laser-driven source development and potential applications, Rev. Sci. Instrum. 87, 071101 (2016).

[4] S. V. Bulanov and V. S. Khoroshkov, Feasibility of using laser ion accelerators in proton therapy, Plasma Phys. Rep. 28, 453 (2002).

[5] M. Passoni, L. Bertagna, and A. Zani, Target normal sheath acceleration: Theory, comparison with experiments and future perspectives, New J. Phys. 12, 045012 (2010).

[6] V. T. Tikhonchuk, A. A. Andreev, S. G. Bochkarev, and V. Y. Bychenkov, Ion acceleration in short-laser-pulse interaction with solid foils, Plasma Phys. Controlled Fusion 47, B869 (2005).

[7] A. Higginson, R. J. Gray, M. King, R. J. Dance, S. D. R. Williamson, N. M. H. Butler, R. Wilson, R. Capdessus, C. Armstrong, J. S. Green et al., Near-100 mev protons via a laser-driven transparency-enhanced hybrid acceleration scheme, Nat. Commun. 9, 724 (2018).

[8] S. Kar, K. F. Kakolee, B. Qiao, A. Macchi, M. Cerchez, D. Doria, M. Geissler, P. McKenna, D. Neely, J. Osterholz et al., Ion Acceleration in Multispecies Targets Driven by Intense Laser Radiation Pressure, Phys. Rev. Lett. 109, 185006 (2012).

[9] T. Esirkepov, M. Borghesi, S. V. Bulanov, G. Mourou, and T. Tajima, Highly Efficient Relativistic-Ion Generation in the Laser-Piston Regime, Phys. Rev. Lett. 92, 175003 (2004).

[10] S. V. Bulanov, E. Y. Echkina, T. Z. Esirkepov, I. N. Inovenkov, M. Kando, F. Pegoraro, and G. Korn, Unlimited Ion Acceleration by Radiation Pressure, Phys. Rev. Lett. 104, 135003 (2010).

[11] A. Macchi, S. Veghini, and F. Pegoraro, "Light Sail" Acceleration Reexamined, Phys. Rev. Lett. 103, 085003 (2009).

[12] A. Sgattoni, S. Sinigardi, and A. Macchi, High energy gain in three-dimensional simulations of light sail acceleration, Appl. Phys. Lett. 105, 084105 (2014).

[13] A. P. L. Robinson, M. Zepf, S. Kar, R. G. Evans, and C. Bellei, Radiation pressure acceleration of thin foils with 
circularly polarized laser pulses, New J. Phys. 10, 013021 (2008).

[14] X. Zhang, B. Shen, X. Li, Z. Jin, F. Wang, and M. Wen, Efficient $\mathrm{GeV}$ ion generation by ultraintense circularly polarized laser pulse, Phys. Plasmas 14, 123108 (2007).

[15] O. Klimo, J. Psikal, J. Limpouch, and V. T. Tikhonchuk, Monoenergetic ion beams from ultrathin foils irradiated by ultrahigh-contrast circularly polarized laser pulses, Phys. Rev. ST Accel. Beams 11, 031301 (2008).

[16] C. Scullion, D. Doria, L. Romagnani, A. Sgattoni, K. Naughton, D. Symes, P. McKenna, A. Macchi, M. Zepf, S. Kar et al., Polarization Dependence of Bulk Ion Acceleration from Ultrathin Foils Irradiated by High-Intensity Ultrashort Laser Pulses, Phys. Rev. Lett. 119, 054801 (2017).

[17] A. Henig, S. Steinke, M. Schnürer, T. Sokollik, R. Hörlein, D. Kiefer, D. Jung, J. Schreiber, B. M. Hegelich, X. Q. Yan et al., Radiation-Pressure Acceleration of Ion Beams Driven by Circularly Polarized Laser Pulses, Phys. Rev. Lett. 103, 245003 (2009).

[18] J. Bin, W. Ma, H. Wang, M. Streeter, C. Kreuzer, D. Kiefer, M. Yeung, S. Cousens, P. Foster, B. Dromey et al., Ion Acceleration using Relativistic Pulse Shaping in NearCritical-Density Plasmas, Phys. Rev. Lett. 115, 064801 (2015).

[19] X. F. Shen, B. Qiao, H. Zhang, S. Kar, C. T. Zhou, H.X. Chang, M. Borghesi, and X. T. He, Achieving Stable Radiation Pressure Acceleration of Heavy Ions via Successive Electron Replenishment from Ionization of a High- $z$ Material Coating, Phys. Rev. Lett. 118, 204802 (2017).

[20] X. F. Shen, B. Qiao, H. X. Chang, W. L. Zhang, H. Zhang, C. T. Zhou, and X.T. He, Maintaining stable radiation pressure acceleration of ion beams via cascaded electron replenishment, New J. Phys. 19, 033034 (2017).

[21] B. Qiao, M. Zepf, M. Borghesi, B. Dromey, M. Geissler, A. Karmakar, and P. Gibbon, Radiation-Pressure Acceleration of Ion Beams from Nanofoil Targets: The Leaky Light-Sail Regime, Phys. Rev. Lett. 105, 155002 (2010).

[22] A. Mcllvenny, H. Ahmed, C. Scullion, D. Doria, L. Romagnani, P. Martin, K. Naughton, A. Sgattoni, D. R. Symes, A. Macchi, P. McKenna, M. Zepf, S. Kar, and M. Borghesi, Characteristics of ion beams generated in the interaction of ultra-short laser pulses with ultra-thin foils, Plasma Phys. Controlled Fusion 62, 054001 (2020).

[23] T.-P. Yu, A. Pukhov, G. Shvets, and M. Chen, Stable Laser-Driven Proton Beam Acceleration from a TwoIon-Species Ultrathin Foil, Phys. Rev. Lett. 105, 065002 (2010).

[24] W. J. Ma, I. J. Kim, J. Q. Yu, I. W. Choi, P. K. Singh, H. W. Lee, J. H. Sung, S. K. Lee, C. Lin, Q. Liao, J. G. Zhu, H. Y. Lu, B. Liu, H. Y. Wang, R. F. Xu, X. T. He, J. E. Chen, M. Zepf, J. Schreiber, X. Q. Yan, and C. H. Nam, Laser Acceleration of Highly Energetic Carbon Ions using a Double-Layer Target Composed of Slightly Underdense Plasma and Ultrathin Foil, Phys. Rev. Lett. 122, 014803 (2019).

[25] C. Hooker, Y. Tang, O. Chekhlov, J. Collier, E. Divall, K. Ertel, S. Hawkes, B. Parry, and P. Rajeev, Improving coherent contrast of petawatt laser pulses, Opt. Express 19, 2193 (2011).

[26] G. Milluzzo, H. Ahmed, L. Romagnani, D. Doria, P. Chaudhary, C. Maiorino, A. McIlvenny, A. McMurray, K. Polin, Y. Katzir, R. Pattathil, P. McKenna, K. Prise, and M. Borghesi, Dosimetry of laser-accelerated carbon ions for cell irradiation at ultra-high dose rate, J. Phys. 1596, 012038 (2020).

[27] B. Dromey, S. Kar, M. Zepf, and P. Foster, The plasma mirror-a subpicosecond optical switch for ultrahigh power lasers, Rev. Sci. Instrum. 75, 645 (2004).

[28] A. Lévy, T. Ceccotti, P. D’Oliveira, F. Réau, M. Perdrix, F. Quéré, P. Monot, M. Bougeard, H. Lagadec, P. Martin, J.-P. Geindre, and P. Audebert, Double plasma mirror for ultrahigh temporal contrast ultraintense laser pulses, Opt. Lett. 32, 310 (2007).

[29] D. Wang, Y. Shou, P. Wang, J. Liu, Z. Mei, Z. Cao, J. Zhang, P. Yang, G. Feng, S. Chen et al., Laser-induced damage thresholds of ultrathin targets and their constraint on laser contrast in laser-driven ion acceleration experiments, High Power Laser Sci. Eng. 8, e41 (2020).

[30] A. Henig, D. Kiefer, K. Markey, D. C. Gautier, K. A. Flippo, S. Letzring, R. P. Johnson, T. Shimada, L. Yin, B. J. Albright, K. J. Bowers, J. C. Fernández, S. G. Rykovanov, H.-C. Wu, M. Zepf, D. Jung, V. K. Liechtenstein, J. Schreiber, D. Habs, and B. M. Hegelich, Enhanced Laser-Driven Ion Acceleration in the Relativistic Transparency Regime, Phys. Rev. Lett. 103, 045002 (2009).

[31] N. P. Dover et al., Buffered high charge spectrally-peaked proton beams in the relativistic-transparency regime, New J. Phys. 18, 013038 (2016).

[32] S. Steinke, P. Hilz, M. Schnürer, G. Priebe, J. Bränzel, F. Abicht, D. Kiefer, C. Kreuzer, T. Ostermayr, J. Schreiber, A. A. Andreev, T. P. Yu, A. Pukhov, and W. Sandner, Stable laser-ion acceleration in the light sail regime, Phys. Rev. ST Accel. Beams 16, 011303 (2013).

[33] T. D. Arber, K. Bennett, C. S. Brady, A. Lawrence-Douglas, M. G. Ramsay, N. J. Sircombe, P. Gillies, R. G. Evans, H. Schmitz, A. R. Bell, and C. P. Ridgers, Contemporary particle-in-cell approach to laser-plasma modelling, Plasma Phys. Controlled Fusion 57, 113001 (2015).

[34] L. Obst, J. Metzkes-Ng, S. Bock, G. E. Cochran, T. E. Cowan, T. Oksenhendler, P. L. Poole, I. Prencipe, M. Rehwald, C. Rödel, H.-P. Schlenvoigt, U. Schramm, D. W. Schumacher, T. Ziegler, and K. Zeil, On-shot characterization of single plasma mirror temporal contrast improvement, Plasma Phys. Controlled Fusion 60, 054007 (2018).

[35] J. Posthumus, M. Thompson, L. Frasinski, and K. Codling, Molecular dissociative ionisation using a classical over-thebarrier approach, Multiphoton Processes 1996 154, 298 (1997).

[36] N. B. Delone and Kră̌, Multiphoton Processes in Atoms, Springer Series on Atomic, Optical, and Plasma Physics Vol. 13 (Springer-Verlag, Berlin Heidelberg, 1994), https:// link.springer.com/book/10.1007\%2F978-3-642-97509-7.

[37] The EPOCH code uses the so-called ADK rate from M. V. Ammosov, N. B. Delone, and V. P. Krainov, Tunnel Ionization Of Complex Atoms And Atomic Ions In 
Electromagnetic Field, High Intensity Laser Processes 0664, 138 (1986); Actually the ADK rate can be found in preceding work: Nikishov and Ritus, Sov. Phys. JETP 23, 162 (1966); Perelomov et al., Ionization of atoms in an alternating electric field, Sov. Phys. JETP 23, 924 (1966).

[38] F. Prez, L. Gremillet, A. Decoster, M. Drouin, and E. Lefebvre, Improved modeling of relativistic collisions and collisional ionization in particle-in-cell codes, Phys. Plasmas 19, 083104 (2012).
[39] See Supplemental Material at http://link.aps.org/supplemental/ 10.1103/PhysRevLett.127.194801 for additional information on the experimental and simulation data.

[40] T. Ziegler et al., Proton beam quality enhancement by spectral phase control of a PW-class laser system, Sci. Rep. 11, 7338 (2021).

[41] QUB Link: https://doi.org/10.17034/8680610b-5046-47cfae2c-a713ac2a0645. 